\title{
HER2-pulsed Autologous Type-1 Polarized Dendritic Cell Vaccine
}

National Cancer Institute

\section{Source}

National Cancer Institute. HER2-pulsed Autologous Type-1 Polarized Dendritic Cell

Vaccine. NCl Thesaurus. Code C114293.

A dendritic cell (DC)-based cancer vaccine composed of autologous, type-1 polarized dendritic cells (DCS) pulsed with human leukocyte antigen (HLA)-A2-restricted HER-2derived peptides, with potential immunomodulatory and antineoplastic activities. Autologous DCs were treated with GM-CSF, interleukin-4, interferon-gamma and bacterial lipopolysaccharide (LPS), a toll-like receptor type 4 agonist, to produce highly polarized DCs (alphaDC1) that are capable of producing high levels of interleukin-12p70 (IL-12p70). Upon administration, HER2-pulsed autologous DC vaccine may stimulate a potent cytotoxic T-lymphocyte (CTL) response ag ainst HER-2-positive tumor cells, which may result in tumor cell death and decreased tumor growth. HER-2, a tyrosine kinase receptor for epidermal growth factor (EGF) (also known as neu and ErbB2), is overexpressed by a variety of cancers. 